\title{
Seguridad clínica percibida por los pacientes en diálisis peritoneal
}

\author{
Ma Encarnación Hernández Meca* - Antonio Ochando García* - Catalina Royuela García* - Susana Lo- \\ renzo Martínez** - Fernando Paniagua**
}

Unidad de Diálisis* - Unidad de Calidad** - Fundación Hospital Alcorcón. Madrid

\section{RESUMEN}

Aunar esfuerzos y conseguir el máximo grado de calidad y seguridad en la atención prestada es una responsabilidad de los profesionales sanitarios. Nos propusimos evaluar la percepción de la seguridad clínica en diálisis peritoneal percibida por el paciente y determinar la importancia que tiene la comunicación-información del personal sanitario.

Estudio descriptivo y transversal, a todos los pacientes en diálisis peritoneal, administrando una encuesta de opinión sobre seguridad clínica. Se analizaron 38 encuestas (tasa respuesta $100 \%$ ). El 60.5\% eran hombres y el $39.5 \%$ mujeres. Edad media $59,1 \pm 12$ años y tiempo medio en técnica 24.4 \pm 19.7 meses. El $71 \%$ de los pacientes manifestó que les preocupaba mucho sufrir un error médico. E $100 \%$ de los pacientes que habían sufrido un efecto indeseado como consecuencia de la asistencia o de la medicación administrada, manifestaron estar muy preocupados por sufrir un error médico.

Al analizar las preguntas relacionadas con la información-comunicación entre el personal sanitario y el paciente, el $81 \%$ manifestaron que les habían

Correspondencia:

$M^{a}$ Encarnación Hernández Meca

C/ Rozabellas $n^{\circ} 11$

28290 Las Rozas (Madrid)

mehernandez@fhalcorcon.es explicado, con detalle, los efectos y precauciones del tratamiento y el $84,2 \%$ respondió que habían podido consultar sus dudas y preocupaciones a la enfermera y al médico con la suficiente intimidad. Sin embargo, aquellos pacientes que detectaron un déficit en la información-comunicación en alguno de estos aspectos, comentaron estar muy preocupados por poder sufrir un error médico.

La seguridad clínica suscita interés y preocupación entre los pacientes. La falta de informacióncomunicación se relaciona con una mayor preocupación de sufrir un error médico.

\section{PALABRAS CLAVE:}

- SEGURIDAD CLÍNICA

- DIÁLISIS PERITONEAL

- ENCUESTA

Clinical safety perceived by patients on peritoneal dialysis

\section{Abstract}

Combining efforts and achieving the maximum degree of quality and safety in the care provided is the responsibility of healthcare professionals. We proposed to assess the perception of clinical safety in peritoneal dialysis perceived by the patient and to determine the importance of communication-information provided by healthcare personnel. 
Descriptive transversal study, encompassing all patients on peritoneal dialysis, distributing a survey seeking their opinion on clinical safety. 38 surveys were analysed (response rate 100\%). $60.5 \%$ were men and $39.5 \%$ women. Average age $59.1 \pm 12$ years and average time on the dialysis technique $24.4 \pm 19.7$ months. $71 \%$ of the patients stated that they were very concerned about suffering a medical error. $100 \%$ of the patients who had suffered an undesired effect as a result of the care or medication administered stated that they were very worried about medical errors.

On analysing the questions relating to informationcommunication between healthcare personnel and the patient, $81 \%$ indicated that the effects and precautions of the treatment had been explained to them in detail, and $84.2 \%$ responded that they had been able to discuss their doubts and concerns with the nurse and doctor in sufficient privacy. However, those patients who detected a deficit in the information-communication in any of these aspects mentioned that they were very concerned about suffering from a medical error.

Clinical safety arouses interest and concern among patients. The lack of information-communication is related to greater concern about suffering from a medical error.

\section{KEY WORDS:}

CLINICAL SAFETY

- PERITONEAL DIALYSIS

\section{SURVEY}

\section{Introducción}

El concepto de seguridad de los pacientes como componente clave de la calidad, se originó en el mundo de la industria y de los servicios y, se ha ido introduciendo y adaptando durante los años 90 a la realidad del sector de la salud de los países desarrollados. Hoy día forma parte de las estrategias de calidad de muchos países . El Ministerio de Sanidad y Consumo, al reconocer esta realidad, ha realizado una línea de trabajo estratégica sobre la seguridad del paciente que está en consonancia con la de la Organización Mundial de la
Salud (OMS), que recientemente anunció la puesta en marcha de la Alianza Mundial para la Seguridad del Paciente ${ }^{1}$.

Es responsabilidad de los profesionales sanitarios, entre otros, el aunar esfuerzos y conseguir el máximo grado de calidad y seguridad en la atención prestada, para ello, es crucial introducir un cambio de cultura que nos lleve a aprender de los errores y a controlarlos; $y$, en consecuencia, ofrecer a los pacientes unos servicios de calidad crecientes.

La información adecuada e interactiva, constituye el eje sobre el que gravita la percepción de seguridad del paciente. La correcta gestión de la incertidumbre en la relación paciente- profesional mejora la seguridad, construye un entorno de confianza y genera satisfacción en el paciente?

Son múltiples las publicaciones que abordan los riesgos hospitalarios, sin embargo, son escasas las investigaciones que estudian los eventos adversos en las unidades de diálisis ${ }^{3}$, así como aquellas que estudian estos aspectos desde la percepción de los pacientes.

En el mundo de la Nefrología se ha producido un importante avance a nivel tecnológico. Los profesionales que trabajamos en este ámbito sabemos que la seguridad de los equipos es cada vez mayor, los tratamientos más complejos y que el perfil del paciente ha cambiado; demanda más información sobre su tratamiento y quiere ser participe de lo que le acontece en todo momento. La Ley de Autonomía del paciente, derechos y deberes $^{4}$ avala la importancia de la comunicación, algo tan simple y complejo a la vez ${ }^{5}$.

\section{Objetivos}

- Evaluar la percepción de los pacientes en diálisis peritoneal sobre la seguridad clínica.

- Determinar la importancia que tiene la comunicación-información del personal sanitario sobre la seguridad percibida por los pacientes.

\section{Material y Método}

Periodo de estudio: El presente estudio se ha desarrollado durante los meses de febrero y marzo de 2007. 
Estudio: Se ha realizado un estudio observacional, descriptivo, de corte transversal a todos los pacientes en diálisis peritoneal de nuestro hospital.

Ámbito: Unidad de diálisis peritoneal de la Fundación Hospital Alcorcón. Hospital público nivel III de la Comunidad Autónoma de Madrid, con una población de referencia en el área de nefrología de 550.000 habitantes. Se informó a los pacientes sobre el objetivo del estudio, solicitando su consentimiento verbal, previo a la entrega de una encuesta; se facilitó la información e instrucciones necesarias para su correcta cumplimentación, depositándola en un buzón colocado al efecto en la unidad, garantizando en todo momento el anonimato. De los 49 pacientes en tratamiento con diálisis peritoneal en nuestro hospital, sólo se pudo entregar un total de 38 cuestionarios, ya que 2 pacientes fueron trasplantados y 9 pacientes no acudieron a revisión durante el periodo de estudio.

Instrumento de evaluación: Para medir la seguridad percibida por el paciente se utilizó una encuesta de opinión sobre seguridad clínica (anexo 1), en cuya elaboración participó el gabinete de calidad de la Fundación Hospital Alcorcón.

Anexo 1

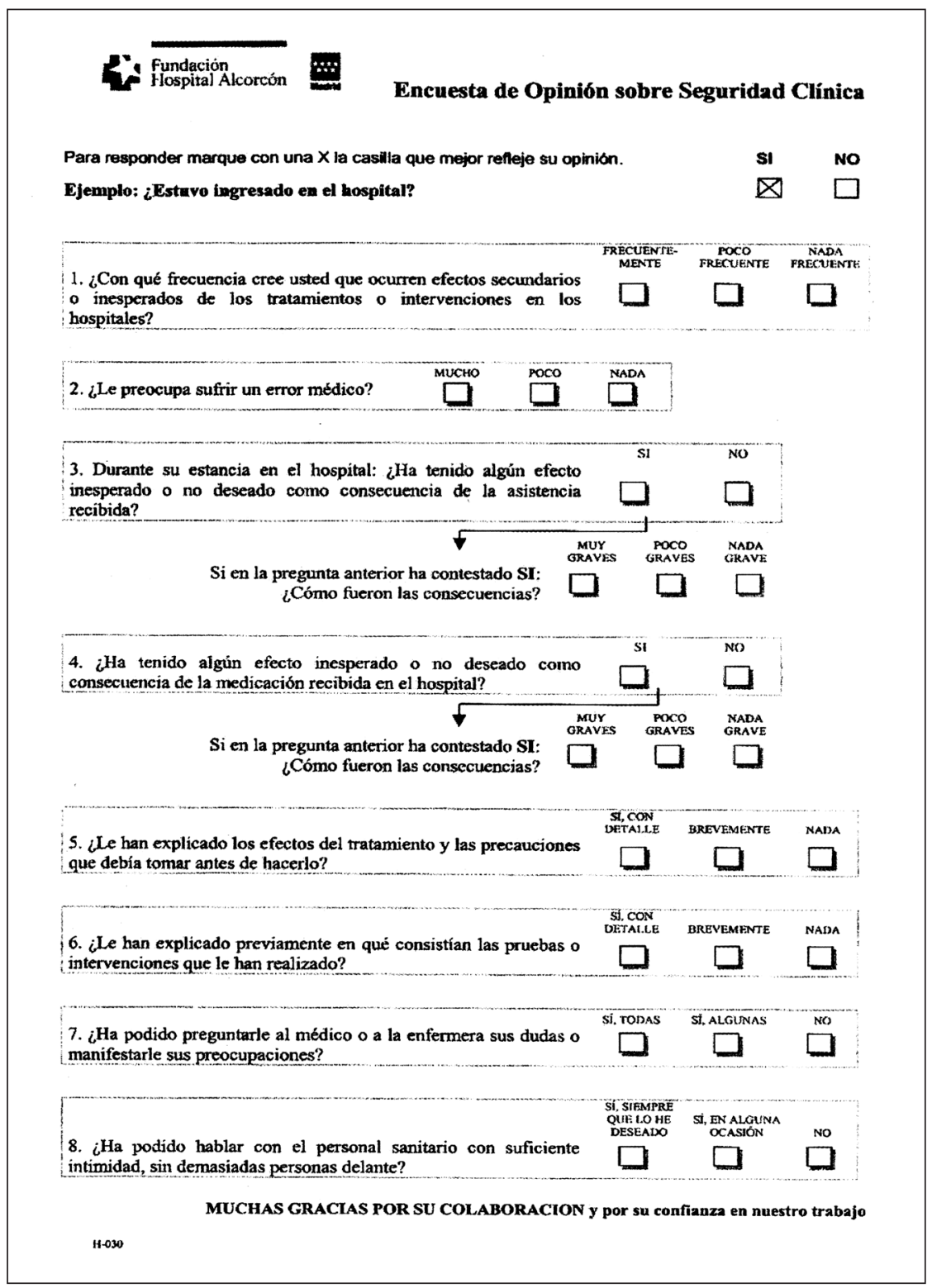


La encuesta consta de 8 ítems, con tres opciones de respuesta, en los que se aborda la percepción de los errores en la asistencia sanitaria, así como la información y la comunicación entre el personal sanitario y el paciente. También se recogieron las características sociodemográficas de los pacientes.

Análisis estadístico: Una vez recogidos los cuestionarios, toda la información se introdujo en una base de datos ad-hoc, volcando estos datos en el programa estadístico SPSS 12.0 para Windows. Se realizó un análisis descriptivo de la frecuencia y media de respuesta en cada una de las preguntas.

\section{Resultados}

Se entregaron 38 cuestionarios de los que se recogieron 38, es decir hubo una tasa de respuesta del $100 \%$. El $60,5 \%$ de los pacientes eran hombres y el $39,5 \%$ mujeres. La edad media era de 59,1 \pm 12 años y un tiempo medio en técnica de $24,4 \pm 19,7$ meses.

Al preguntar a los pacientes con qué frecuencia creen ellos que ocurren efectos secundarios o inesperados en los tratamientos o intervenciones realizadas, el $64,86 \%$ contestó que con "poca frecuencia", el 18,9 contestó "frecuentemente" y el 16,22\% "nada frecuente".

Referente a la pregunta ¿les preocupa sufrir errores médicos?", el $71 \%$ de los pacientes contestó "mucho", el 15,7\% "poco" y el 13,1\% "nada". Treinta y cuatro pacientes $(89,4 \%)$ respondieron no haber sufrido ningún efecto inesperado de la asistencia recibida, mientras que $4(10,53 \%)$ pacientes si manifestaron haber sufrido efectos adversos, de éstos, 1 paciente manifiesto haber sufrido consecuencias "muy graves", 2 pacientes "poco graves" y 1 paciente "nada graves".

Referente a la administración de la medicación, la situación es igual a la de los efectos adversos en la asistencia recibida, es decir, 34 pacientes no sufrieron ningún efecto adverso como consecuencia de la medicación, 1 paciente manifestó haber sufrido consecuencias muy graves, 2 pacientes "poco graves" y 1 paciente "nada graves" (figura 1 ).

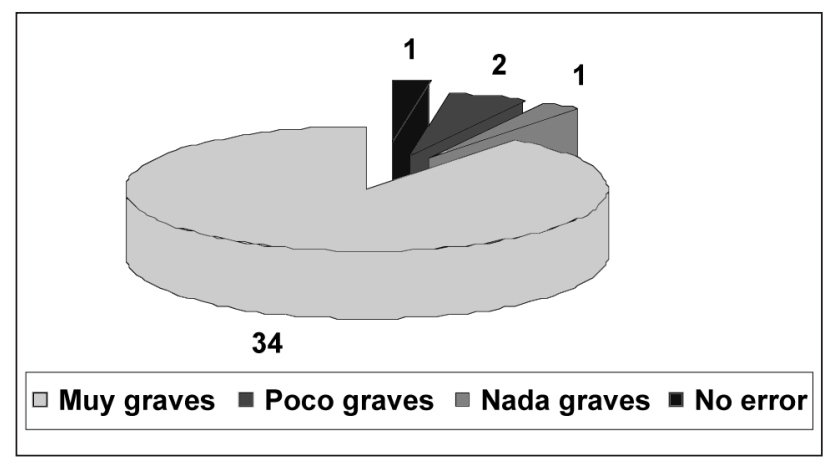

Figura 1: Efectos adversos sufridos en la asistencia recibida y en la medicación administrada

Al analizar las preguntas relacionadas con la información y la comunicación entre el personal sanitario y el paciente (figura 2), el $81 \%$ piensan que les fue explicado "con detalle" los efectos y precauciones del tratamiento, el 16,2\% "brevemente" y el 2,7\% piensan que no les habían explicado "nada". A la pregunta si les habían explicado en qué consistían las pruebas e intervenciones a realizar, el $89,1 \%$ piensan que fueron explicadas "con detalle", el 8,1\% "brevemente" y el 2,7\% "nada".

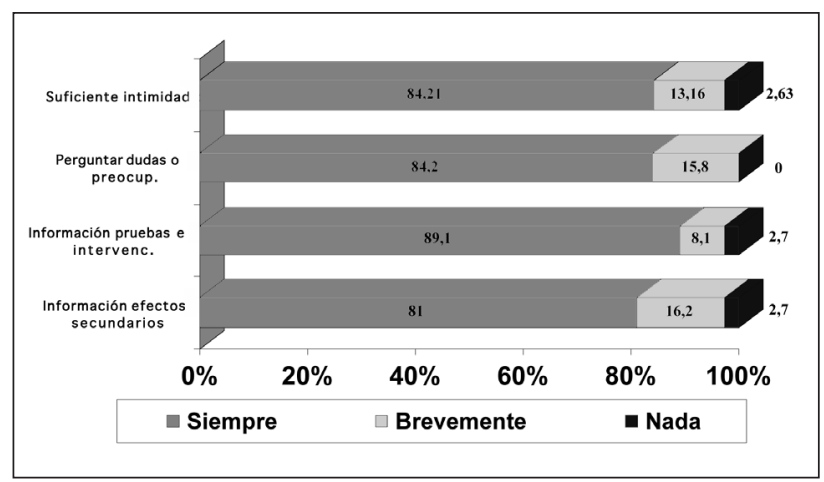

Figura 2: Información y comunicación con el paciente

A la pregunta que evaluaba si habían podido manifestar sus dudas o preocupaciones al médico o a la enfermera, el $84,2 \%$ respondió "sí, todas" y el $15,8 \%$ "sí, algunas". No hubo ningún paciente que no hubiera podido manifestar sus preocupaciones 0 dudas. El 84,21\% de los pacientes encuestados respondió que "siempre que lo he deseado" había podido hablar con el personal sanitario con suficiente intimidad y sin demasiadas personas delante, el $13,16 \%$ contestó "sí, en alguna ocasión" y el 2,63\% respondió "no". 
Todos los pacientes que habían sufrido un efecto indeseado como consecuencia de la asistencia recibida o habían sufrido un efecto adverso como consecuencia de la medicación administrada, independientemente de si las consecuencias eran muy graves, poco graves o nada graves, manifestaron estar muy preocupados por sufrir un error médico. También respondieron estar muy preocupados por poder sufrir un error médico, los pacientes que:

- manifestaron no haber podido hablar con intimidad con el personal sanitario en todas las ocasiones que lo hubieran deseado (6 pacientes)

- no habían podido preguntar todas sus dudas con detalle al médico o a la enfermera (6 pacientes)

- no habían recibido información detallada de las pruebas que les iban a realizar (4 pacientes)

- no habían recibido información de los efectos del tratamiento y precauciones del mismo (6 pacientes).

\section{Discusión}

En el sistema sanitario actual el paciente se ha convertido en centro y motor del mismo ${ }^{6}$. En nuestro estudio, el alto grado de participación de los pacientes (tasa de respuesta del 100\%), de forma voluntaria y anónima, nos muestra la importancia que tiene para ellos el que nos preocupemos en conocer su opinión.

En la actualidad encontramos múltiples publicaciones que abordan la seguridad clínica desde el punto de vista de las instituciones-personal sanitario, sin embargo con este trabajo hemos querido recoger la opinión de los pacientes con relación a este aspecto.

En las últimas décadas el perfil del paciente con enfermedad renal crónica está cambiando, nos encontramos con un paciente de mayor edad, con más patologías, y por tanto con una importante demanda asistencial. En el momento actual, la DP está consolidada como la técnica dialítica domiciliaria preferente, que los pacientes escogen como primera opción de diálisis en un porcentaje apreciable. La DP en cualquiera de sus modalidades, Diálisis Peritoneal Continua Ambulatoria o Diálisis Peritoneal Automatizada, potencia la autonomía del paciente, con buenos resultados de integración social y de satisfacción personal, evitando el incremento de inversión en infraestructuras ${ }^{7}$.

El contar con la opinión de los pacientes nos ayuda a la hora de elaborar posibles estrategias de mejora en la atención sanitaria, no podemos olvidar que la seguridad clínica, junto a la satisfacción, la utilización eficiente de los recursos, entre otros, es uno de los aspectos que van íntimamente ligados a la calidad asistencial ${ }^{8}$.

Como han hallado otros autores, la comunicación, real y efectiva, entre profesional y paciente es uno de los aspectos fundamentales para valorar el nivel de seguridad del paciente?.

En todas las preguntas relativas a la información y comunicación entre el personal sanitario y el paciente, más del $80 \%$ de los pacientes manifestaron: haber recibido información detallada sobre su tratamiento y/o las pruebas que se le iban a realizar, habían podido preguntar al personal sanitario todas sus dudas y/o preocupaciones y habían tenido la intimidad que habían necesitado. Sin embargo encontramos que todos aquellos pacientes ( $100 \%$ ) que no habían podido consultar sus dudas al personal sanitario, o no habían contado con la intimidad que les hubiera gustado, o no recibieron información detallada sobre su tratamiento o las pruebas o intervenciones prescritas, tenían una preocupación mayor de sufrir un error médico.

\section{Conclusiones}

- La seguridad clínica es un tema que suscita interés y preocupación entre los pacientes en diálisis peritoneal de nuestra unidad.

- La falta de información-comunicación por parte del personal sanitario se relaciona con una mayor preocupación de sufrir un error médico, en nuestros pacientes de diálisis peritoneal. 


\section{Bibliografía}

1. Salgado E. Prólogo. Rev. Calidad Asistencial, 2005; 20 (2): 49-50.

2. Aibar C. La percepción del riesgo: del paciente informado al paciente consecuente. Humanitas: $\mathrm{Hu}$ manidades médicas; 2005. Consultado el 10/02/2007 en: http://www.fundacionmhm.org/pdf/Mono8/Articulos/articul03.pdf

3. Holley J. A descriptive report of errors and adverse events in chronic hemodialysis units. Nephrol News Issues. 2006; 20(12):57-63.

4. Boletín Oficial del Estado. Ley $41 / 2002$ de 14 de noviembre, Básica Reguladora de la Autonomía del Paciente y de Derechos y Obligaciones en Materia de Información y Documentación clínica. BOE de 15/11/2002.
5. Sistemas de registro y notificación de incidentes y eventos adversos. Secretaría General de Sanidad. Agencia de calidad del Sistema Nacional de Salud. Ministerio de Sanidad y Consumo.

6. Diaz R. Satisfacción del paciente: principal motor y centro de los servicios sanitarios. Rev Calidad Asistencial 2002; 17(1):22-29.

7. Guías SEN. Guías de práctica clínica en diálisis peritoneal de la SEN. Nefrología 2006; 26 (4).

8. Lorenzo S, Mira JJ, Garcia EI. Gestión de la calidad, En Ayuso D, Grande RF. La gestión de Enfermería y los servicios generales en las organizaciones sanitarias. Ed. Diaz de Santos. 2006: 527-555.

9. Remón CA. La percepción del riesgo: del paciente informado al paciente consecuente. Monografías humanitas, 2004 - fundacionmhm.org 